\title{
Evidencia de estructura y consistencia interna del Inventario de Riesgo Suicida en Adolescentes (IRISA) uruguayos
}

\section{Evidence of structure and internal consistency of the Risk Inventory Suicidal Adolescent (IRISA) in Uruguay population}

\section{Evidência de Estrutura e Consistência Interna do Inventário de Risco Suicida (IRISA) em Adolescentes Uruguaios}

\author{
Ana Inés Machado ${ }^{1}$, ORCID: 0000-0003-0197-6083 \\ Antonella Bobbio ${ }^{2}$, ORCID 0000-0003-4121-9482 \\ Karin Arbach ${ }^{3}$, ORCID 0000-0003-1753-4693 \\ Alfredo Parra ${ }^{4}$, ORCID 0000-0003-3874-653X \\ Claudia Riestra ${ }^{5}$, ORCID 0000-0002-8662-4898 \\ Quetzalcoátl Hernández-Cervantes ${ }^{6}$, ORCID 0000-0002-9728-5406 \\ ${ }^{15}$ Universidad Católica del Uruguay \\ ${ }^{2} 3$ Universidad Nacional de Córdoba, Argentina \\ ${ }^{4}$ Universidad de la República, Uruguay \\ ${ }^{6}$ Universidad Iberoamericana Puebla, México
}

Resumen: Uruguay ha registrado un incremento del $40 \%$ en los suicidios durante los últimos 15 años; el $17 \%$ de quienes cometen estas conductas son adolescentes. Ante la importancia de contar con instrumentos adecuados para valorar y manejar el riesgo suicida en esta etapa, el presente estudio analiza algunas propiedades psicométricas del Inventario de Riesgo Suicida para Adolescentes (IRISA). A una muestra de 447 estudiantes uruguayos, con un rango de edad de entre 12 y 18 años $(M=15.06 ; D E=1.84)$, se les administró un cuestionario sociodemográfico y el IRISA. El análisis factorial confirmatorio corroboró la dimensionalidad de la escala, los adecuados índices de fiabilidad y las correlaciones esperables intra-subescalas. Se concluye que el IRISA muestra propiedades psicométricas adecuadas, por lo que constituye una herramienta útil para la identificación de aquellos adolescentes en riesgo suicida y su uso extensivo en los servicios de atención primaria de la salud podría resultar prometedor.

Palabras clave: riesgo suicida; adolescencia; IRISA; estudio instrumental

Abstract: Suicide rates have risen by $40 \%$ over the last 15 years in Uruguay; $17 \%$ of people who commit suicide in Uruguay are adolescents. Acknowledging the need for appropriate instruments to assess and manage suicidal risk among adolescents, this study evaluated some psychometric properties of the Adolescent Suicide Risk Inventory (IRISA, acronym in Spanish). The IRISA and a sociodemographic questionnaire were administered to a sample of Uruguayan adolescent students of 447 individuals, aged 12 through $18(M=15.06 ; S D=1.84)$. The confirmatory factorial analysis concurs with the reported scales of IRISA, exhibiting adequate reliability and acceptable inter-scale correlations. Findings suggest IRISA is a valid and reliable screening test for suicidal risk in Uruguayan student adolescents and its extensive use at primary care service seems promising. 
Keywords: suicide risk; adolescence; IRISA; instrumental study

Resumo: Nos últimos 15 anos o Uruguai registrou um aumento de $40 \%$ nos casos de suicídio. Deste número, $17 \%$ são adolescentes. Diante da importância de contar com instrumentos adequados para avaliar e prevenir o risco de suicídio nesta etapa, o presente estudo analisa algumas propriedades psicométricas do Inventário de Risco Suicida para Adolescentes (IRISA). Em uma amostra de 447 adolescentes uruguaios, com uma faixa etária entre 12 e 18 anos $(M=15.06 ; D E=1.84)$, foi administrado um questionário sociodemográfico e o IRISA. A análise fatorial confirmatória corroborou as dimensões da escala, os adequados índices de confiabilidade e as correlações esperadas intra subescalas. Portanto, se conclui que o IRISA conta com propriedades psicométricas adequadas para a amostra do estudo, já que representa uma ferramenta útil para a identificação daqueles adolescentes com risco de suicídio e seu uso extensivo aos serviços de atenção primária à saúde poderia resultar promissor.

Palavras-chave: risco de suicídio; adolescência; IRISA; estudo instrumental

Recibido: 14/07/2020

Aceptado: 22/03/2021

Cómo citar:

Machado, A. I., Bobbio, A., Arbach, K., Parra, A., Riestra, C. \& Hernández-Cervantes, Q. (2021). Evidencia de estructura y consistencia interna del Inventario de Riesgo Suicida en Adolescentes (IRISA) uruguayos. Ciencias Psicológicas, 15(1), e-2214. doi:

https://doi.org/10.22235/cp.v15i1.2214

Correspondencia: Ana Inés Machado. Departamento de Psicología Clínica y de la Salud, Facultad de Ciencias de la Salud, Universidad Católica del Uruguay. E-mail: amachado@ucu.edu.uy

En la actualidad, el suicidio constituye uno de los problemas de salud pública más preocupantes a nivel mundial. Según datos de la Organización Mundial de la Salud (OMS, 2014), más de 800.000 personas se suicidan cada año en el mundo, lo que significa una muerte cada 40 segundos. Además, anualmente, entre 10 y 20 millones de personas intentan suicidarse. Se estima que, a nivel mundial, en el año 2020 el suicidio representó el 2,4\% del total de la carga de morbilidad; asimismo, la tasa de suicidios ha aumentado en los últimos 50 años aproximadamente un $60 \%$ (OMS, 2012).

Uruguay se ubica entre las 20 naciones con mayores tasas de suicidio a nivel global y es la tercera en América según la Organización Panamericana de la Salud (OPS, 2014). Mientras que en Latinoamérica la prevalencia de suicidios se ha establecido en 7.3, en Uruguay es de 20.25 (Ministerio de Salud Pública [MSP], 2018). Según el MSP (2013), a lo largo de los últimos 15 años se ha producido un incremento de aproximadamente el $40 \%$ de suicidios en el país, lo que representa la muerte de algo más de una persona por día (2018). Como respuesta, el MSP ha implementado mejoras sustanciales en el acceso a la atención universal en salud mental, así como un Plan Nacional de Prevención del Suicidio durante el período 2011-2015, aunque éste no ha alcanzado el impacto esperado respecto al descenso en las cifras de muerte por suicidio. 
La adolescencia es una etapa en la que comúnmente se presentan varias conductas de riesgo, incluidas los intentos de autoeliminación y el suicidio consumado (Sarchiapone, D’Aulerio \& Iosue, 2015). El suicidio es la segunda causa de muerte entre jóvenes de 15 a 29 años (OMS, 2019), y en los últimos 20 años se ha incrementado un $30 \%$ a nivel mundial (Miron, Yu, Wilf-Miron \& Kohane, 2019). Si se considera solamente Hispanoamérica, Uruguay es el segundo país con mayor tasa de suicidios en adolescentes (Ardiles-Irrazábal, Alfaro-Robles, Díaz-Mancilla \& Martínez-Guzmán, 2018). En Uruguay, aproximadamente 42 adolescentes se quitan la vida cada año (OMS, 2018); se estima que 1 de cada 5 muertes entre los 14 y 24 años de edad es por suicidio (MSP, 2013), y 1 de cada 10 adolescentes ha intentado suicidarse. Este grupo etario se define como de alto riesgo para diversas manifestaciones de la violencia, incluida el suicidio (Valdéz, 2010).

El suicidio adolescente presenta algunas particularidades que dificultan su estudio, en comparación con población adulta (Sarchiapone et al., 2015). Por ejemplo, se estima que los datos sobre el comportamiento suicida en la adolescencia se ubican por debajo de su ocurrencia real, dado que muchas muertes por suicidio se catalogan erróneamente como no intencionales o accidentales (OMS, 2014). Adicionalmente, si bien el concepto de muerte, inherente a la intencionalidad suicida, requiere la noción de irreversibilidad, generalmente ya instalada en dicha etapa, ésta puede experimentarse de forma difusa (MSP; Fondo de Población de Naciones Unidas [UNFPA], 2015).

Se concibe al riesgo suicida como un continuum que abarca desde la ideación suicida en sus diferentes manifestaciones, gestos y amenazas suicidas, hasta la planeación del suicidio, los intentos de autoeliminación y el suicidio consumado (OPS, 2014). La presencia de cualquiera de estos comportamientos ya sea de forma esporádica o frecuente, es indicadora de alto riesgo para la persona que los presenta. Considerando que durante la adolescencia se presenta una gran cantidad de conductas de riesgo, algunas asociadas al deseo de morir, su detección y prevención resultan prioritarias.

La prevención del suicidio, como estrategia de salud pública, implica no solamente la detección de intentos de autoeliminación o de psicopatología asociada al comportamiento suicida, sino la sistematización de herramientas que permitan la detección temprana del riesgo suicida en adolescentes. Estas herramientas deberían aplicarse mayoritariamente en los escenarios cotidianos donde los jóvenes lleven a cabo sus actividades principales, como las escuelas o los servicios de atención primaria de salud. Pese a la necesidad de contar con instrumentos que evalúen el riesgo suicida en jóvenes latinoamericanos, las pruebas creadas específicamente, o adaptadas para alcanzar dicho objetivo, son escasas en contextos latinoamericanos. Aunque esto se ha intentado revertir en los últimos años, dichos instrumentos no han demostrado niveles de confiabilidad y validez apropiados (Quinlan-Davidson, Sanhueza, Espinosa, Escamilla-Cejudo \& Maddaleno, 2014). En Uruguay, la mayoría de los instrumentos utilizados en el contexto clínico es originaria de otros entornos socioculturales, y posteriormente traducida al español, sin las adaptaciones correspondientes, lo que conlleva a importantes dificultades comprensivas y lingüísticas al momento de su uso (Villacieros, Bermejo, Magaña \& Fernández-Quijano, 2016).

Entre los instrumentos de valoración de riesgo suicida en muestras latinoamericanas se encuentran: el Inventario de Orientaciones Suicidas (ISO-30; King \& Kowalchuk, 1994, adaptación de Fernández-Liporace \& Casullo, 2006); el Inventario de Razones para Vivir (Echavárri et al., 2018); el Inventario de Ideación Suicida Positiva y Negativa (Pansi; Villalobos-Galvis, 2010); el Inventario de Resiliencia ante el Suicidio (SRI-25; validado por Villalobos-Galvis, Arévalo Ojeda \& Rojas, 2012); la Escala de Columbia de Severidad de Suicidio (C-SSRS, 2011; adaptación de SerraniAzcurri, 2017); la Escala Argentina de Suicidabilidad (Galarza, Castañeiras \& Fernández, 2014); el Inventario de Reconocimiento de Signos de Alerta para Actos Suicidas (IRSA; Brás, Carmo \& Neves de Jesús, 2017) y el Inventario de Riesgo Suicida para Adolescentes en su segunda versión (IRISA; Hernández-Cervantes \& Lucio, 2006). Tras una búsqueda bibliográfica, no se han identificado en 
Uruguay investigaciones que evalúen las propiedades psicométricas de ninguno de estos instrumentos en adolescentes uruguayos.

La mayor parte de los instrumentos mencionados ha puesto un especial énfasis en los factores exclusivamente individuales asociados al riesgo suicida adolescente. El IRISA (Hernández-Cervantes \& Lucio, 2006) es una excepción, pues indaga tanto factores de riesgo como de protección del suicidio, individuales y socio-familiares; evalúa además los distintos componentes del proceso de riesgo suicida adolescente, para estimar el nivel de riesgo suicida del adolescente y proponer un plan terapéutico acorde. Por último, el IRISA contempla condiciones específicas referidas al ámbito académico, donde tiene lugar gran parte del desarrollo de los adolescentes escolarizados, que son la mayoría en Uruguay. El inventario fue creado con base en dos modelos teóricos: el de suicidio en niños y adolescentes propuesto por Shaffer y Pfeffer (2001) y el de identificación y evaluación de riesgo suicida de Kirk (1993) (como se cita en Hernández-Cervantes \& Lucio, 2010). Ambas perspectivas destacan al riesgo suicida como un fenómeno dinámico y multifactorial, que contempla factores biológicos, genéticos, sociodemográficos, familiares y tempranos, presencia de trastornos mentales, personalidad y estilos cognoscitivos, eventos de vida adversos o estresantes, ideación suicida, intentos de suicidio previos, planes y medios para concretar el suicidio.

El inventario, compuesto por 50 reactivos, categoriza al proceso de riesgo en cuatro subescalas: ideación e intencionalidad suicidas (10 ítems; $\alpha=.92)$, depresión y desesperanza (8 ítems; $\alpha=.86$ ), ausencia de circunstancias protectoras (6 ítems; $\alpha=.82$ ), y malestar psicológico asociado al riesgo suicida (26 ítems; $\alpha=.79$ ), y tres ítems críticos, indicadores de los distintos niveles de riesgo: ideación suicida; plan(es) suicida(s), e intento $(s)$ previo(s). El IRISA se define como un instrumento de cribado y priorización del riesgo suicida en población adolescente.

Respecto a las subescalas, ideación e intencionalidad suicidas comprende el grado de intencionalidad para la conducta autolesiva y de la rumiación sobre la propia muerte, en términos de frecuencia y de situaciones comúnmente encontradas en las personas con un alto nivel de planeación (ej., tengo un lugar donde me gustaría morir). Depresión y desesperanza alude al conjunto de cogniciones y afectos negativos respecto a sí mismo, a lo demás y al futuro del adolescente que está siendo examinado, desde los entornos interpersonal, familiar y escolar (ej., la percepción de que no hay cosas buenas en su vida). La tercera subescala, ausencia de circunstancias protectoras, que sigue una inversión en la puntuación y valores de las respuestas, describe el informe que hace el adolescente de la falta de condiciones positivas que previenen la progresión del riesgo, como la cohesión familiar o el vínculo significativo con miembros de su familia o fuera de ella. Finalmente, bajo la subescala de malestar psicológico asociado al riesgo suicida se agrupan tanto situaciones de riesgo como señales de alerta que pueden preceder a las tres áreas anteriormente descritas. Esta es la subescala más amplia en número de ítems y aborda desde dificultades en las materias o asignaturas, con profesores, hasta alteraciones en el dormir o el comer; dichas dificultades no están asociadas exclusivamente al riesgo suicida, pero en combinación con las demás dimensiones convergen en la caracterización de los procesos de riesgo suicida adolescente.

En su primera versión, el IRISA (Hernández-Cervantes \& Lucio, 2003) evaluó población adolescente mexicana en contextos educativos $(N=353)$ y contó con 75 reactivos, que posteriormente se redujeron a 50, para conformar la segunda versión (2006), empleada en el presente estudio. En cuanto a sus propiedades psicométricas, las mismas fueron evaluadas en adolescentes mexicanos escolarizados $(N=555$; Machado, 2006) y se obtuvo evidencia de su validez concurrente tanto con la Escala de Ideación Suicida de Beck (EBIS; González-Macip, Díaz, Ortiz, González \& González, 2000) como con la Subescala Experimental de Ideación Suicida del MMPI-A $(N=221)$ (Lucio, Loza $\&$ Duran, 2000). Además, presentó una consistencia interna $(\alpha=.95)$ y un porcentaje de varianza explicada $(57.6 \%)$ altos. 
Sobre otros estudios réplica que evalúen las propiedades psicométricas del IRISA, solo se encontró una investigación realizada en Colombia $(N=526)$, con una versión abreviada de 24 ítems, cuyos resultados apuntan al inventario como adecuado para evaluar adolescentes en dicho contexto (Alarcón-Vásquez et al., 2019; Bahamón \& Alarcón-Vásquez, 2018). El IRISA mostró buena consistencia a nivel global $(\alpha=.962)$, y en las subescalas, niveles elevados de confiabilidad, que varían entre .798 a .966. El análisis factorial confirmatorio arrojó niveles de ajuste y bondad aceptables. También se identificó la validez convergente con la Escala para la Evaluación del Riesgo Suicida (ERS) de Bahamón y Alarcón-Vásquez (2018). Además, el IRISA ha sido empleado en una muestra normativa paraguaya (Coppari et al., 2009) y en una muestra clínica española, portadora de trastorno adaptativo (Ferrer \& Kirchner, 2014), aunque en ninguno de dichos estudios se realizaron adaptaciones del inventario.

El presente estudio tiene por objetivo evaluar la validez basada en el contenido y la estructura interna, y la consistencia interna del IRISA en una muestra de adolescentes que asisten a escuelas secundarias de cinco departamentos de Uruguay.

\section{Método}

\section{Participantes}

La muestra estuvo conformada por 447 estudiantes (58.16\% mujeres) entre 12 y 18 años $(M$ $=15.06, D E=1.84)$, sin diferencias significativas entre los sexos según la edad, $t(397.6)=-.432, p$ $=.666$. La distribución por cursos tampoco presentó diferencias según sexos, $x^{2}(5, N=447)=2.02$, $p=.846$. Los adolescentes pertenecían a centros educativos correspondientes a cinco departamentos distintos: Montevideo, Canelones, Durazno, Paysandú y Artigas. Los dos primeros departamentos fueron elegidos debido a que poseen la mayor concentración poblacional y de centros educativos, y los otros tres por estar ubicados todos en distintas zonas regionales del país. Hubo un efecto del sexo en la distribución por departamentos $x^{2}(4, N=447)=11.56, p=.021$, con una mayor proporción de varones que de mujeres en Montevideo (42.2\% vs $33.5 \%$ ) y Artigas (12.3\% y $8.1 \%$ ), y a la inversa en el resto de los departamentos. En la Tabla 1 se presentan datos descriptivos sobre las características de la muestra. 
Tabla 1.

Características sociodemográficas de los adolescentes participantes

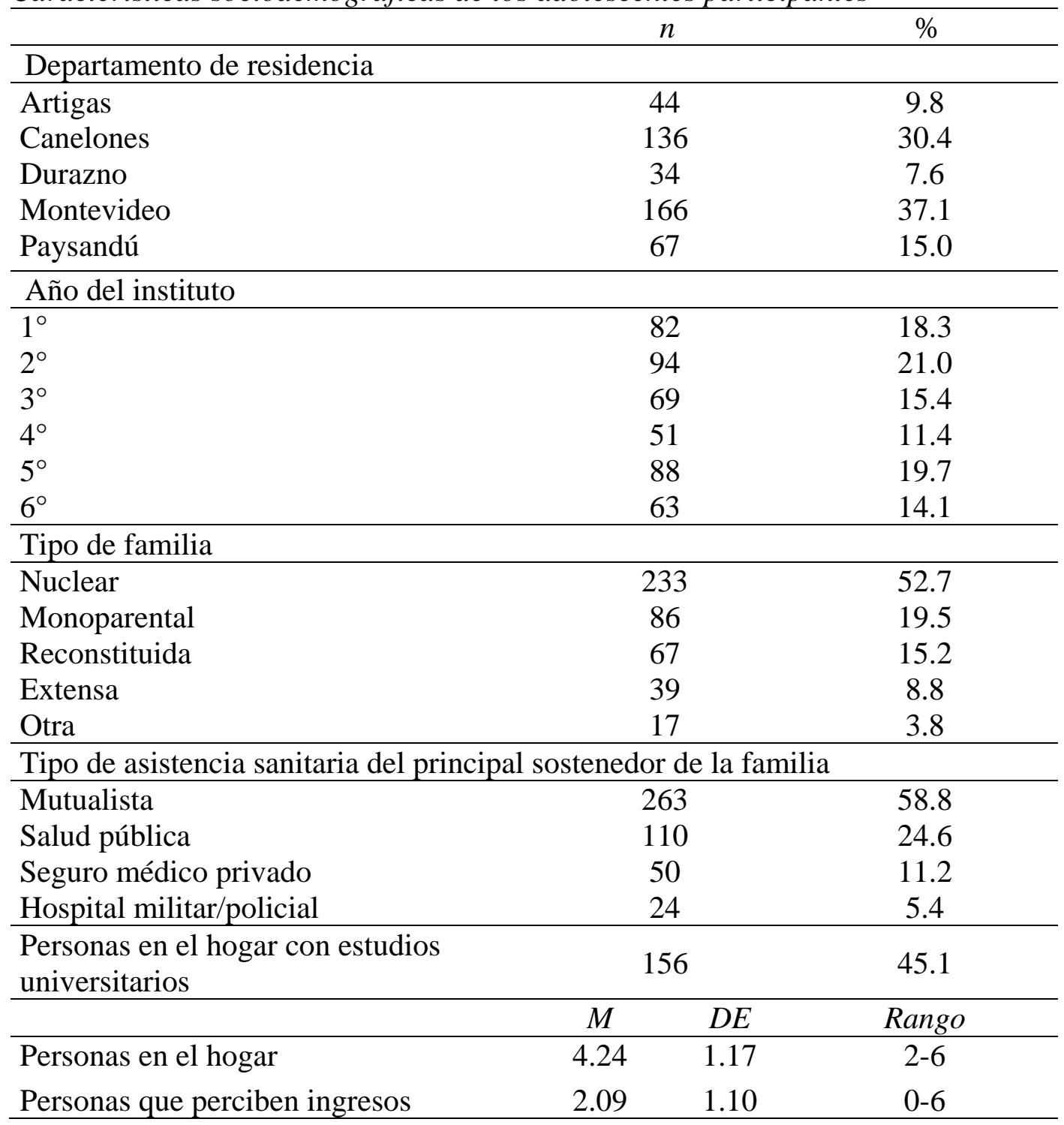

\section{Variables e instrumentos}

Características sociodemográficas. Se empleó una ficha sociodemográfica elaborada ad hoc para evaluar algunos indicadores: departamento de residencia, año de liceo, tipo de familia, tipo de atención sanitaria del principal sostenedor de la familia, personas en el hogar, personas en el hogar con estudios universitarios y personas que perciben ingresos en la familia.

Riesgo suicida. Esta variable se evaluó con el Inventario de Riesgo Suicida para Adolescentes (IRISA; Hernández-Cervantes \& Lucio, 2006). El instrumento está conformado por 50 reactivos que se responden con un formato de respuesta tipo Likert de cinco puntos, 1 (sí, todo este tiempo) a 5 (no, nunca). El tiempo estimado de administración es aproximadamente de 20 minutos.

Como parte de la presente investigación se realizó un análisis de validez de contenido del instrumento en su segunda versión, con metodología no cuantitativa. Para ello, el instrumento original se administró a un grupo de 12 adolescentes de tres rangos de edad: 12-13, 14-15 y 16-18 de ambos sexos, estudiantes de dos centros educativos de distinto nivel socioeconómico (alto y bajo). En cada 
rango se contempló la participación de un adolescente varón y una adolescente mujer, de modo que, en conjunto, en ambas escuelas secundarias se organizaron encuentros con 6 adolescentes, todos ellos a cargo de psicólogos. Los adolescentes consideraron que 46 de los 50 ítems contaban con un alto nivel de claridad y comprensión. En los 4 ítems restantes existieron sugerencias menores de modificación: en 3 de ellos, estas estuvieron vinculadas al uso de los términos escuela y maestros, los cuales fueron sustituidos por liceo y profesores, respectivamente. Un solo ítem fue considerado con mayor dificultad para lograr una equivalencia conceptual (10. El tema de la muerte (mi propia muerte, la de otros, etc.) ha llamado mi atención), y a partir de las sugerencias planteadas en las entrevistas por parte de los adolescentes quedó modificado en: 10. Frecuentemente he pensado sobre la muerte (mi propia muerte, la de otros, etc.). La versión final del inventario adaptado transculturalmente consta de 46 ítems idénticos a la versión utilizada en México, más los 4 que sufrieron modificaciones, solo uno de ellos de contenido más relevante, para un total de 50. Las modificaciones fueron, además, revisadas y aprobadas por una dupla de psicólogos jueces evaluadores, expertos en adolescencia, con el propósito de preservar la transculturalidad para futuras investigaciones empleando el IRISA.

\section{Procedimiento}

El muestreo fue no probabilístico, por conveniencia; el procedimiento para la obtención de la muestra se realizó en instituciones educativas públicas y privadas de Montevideo, Canelones, Durazno, Paysandú y Artigas, quedando integrada por un total de cinco escuelas secundarias. Se consideraron como criterios de inclusión ser adolescente y estar escolarizado en Uruguay. Para realizar las administraciones se solicitó autorización a los directivos de las escuelas secundarias, profesores y padres/tutores. Se obtuvo consentimiento informado verbal de los adolescentes, quienes aceptaron voluntariamente responder al instrumento. La administración de los instrumentos fue grupal y tuvo una duración aproximada de 20 minutos; fue supervisada por psicólogos específicamente capacitados para esta tarea. Cada escuela secundaria participante obtuvo una devolución general de los resultados obtenidos en las áreas de riesgo evaluadas por el IRISA para cada clase evaluada. El presente estudio contó con el aval del Comité de Ética de la Universidad Católica del Uruguay (Nro. de resolución A010317).

\section{Diseño y análisis de datos}

Para el análisis descriptivo se emplearon tablas de distribución de frecuencia y porcentajes. Previo al análisis de las propiedades psicométricas del IRISA se calcularon la media, desviación estándar, simetría y curtosis de cada ítem. Para estos últimos dos índices se consideraron como óptimos los valores entre \pm 1 , y como aceptables los valores entre \pm 2 (George \& Mallery, 2016). Posteriormente se analizaron las correlaciones entre los ítems de cada escala, a fin de detectar problemas de colinealidad. Correlaciones inferiores a .85 se consideraron adecuadas (Cupani, 2012).

Estructura interna. Para evaluar si los datos eran adecuados para llevar a cabo un análisis factorial, se realizaron la prueba Kaiser-Meyer-Olkin (KMO) y el test de esfericidad de Bartlett. En el caso de la prueba KMO, la adecuación de los datos se consideró satisfactoria si los valores superaban .80 y suficientes cuando se encontraban entre .70 y .79 (Lloret-Segura, Ferreres-Traver, Hernández-Baeza \& Tomás-Marco, 2014). El test de esfericidad de Bartlett fue considerado adecuado si presentaba un nivel de significación de $p<.05$ (George \& Mallery, 2016).

Para evaluar los niveles de ajuste del instrumento, se empleó el Análisis Factorial Confirmatorio (AFC). Se optó por emplear el método de estimación de Máxima Verosimilitud Robusto (MLR), dado que trabaja con correlaciones tetracóricas y policóricas, apropiadas para los datos que presentan categorías de respuesta en una escala ordinal, como las del instrumento analizado en este estudio (Freiberg-Hoffmann, Stover, de la Iglesia \& Fernández-Liporace, 2013). El modelo 
fue especificado con base en la estructura factorial propuesta en la segunda versión del instrumento (Hernández-Cervantes \& Lucio, 2006), empleada en este estudio. Para evaluar el ajuste del modelo se emplearon múltiples indicadores: el estadístico chi cuadrado $\left(\chi^{2}\right)$, la razón de chi cuadrado sobre los grados de libertad $\left(\chi^{2} / \mathrm{gl}\right)$, el índice de ajuste comparativo (CFI), el índice de Tucker-Lewis (TLI) y el error cuadrático medio de aproximación (RMSEA). Se esperó que la razón de chi cuadrado sobre los grados de libertad arrojara valores inferiores a 3.0. Para los índices CFI y TLI se consideraron valores de entre .90 y .95 como indicadores de un ajuste aceptable, y superiores a .95 como un ajuste excelente. Para el caso del RMSEA se interpretaron valores de entre .05 y .08 como aceptables o inferiores a .05 como excelentes (Hair, Anderson, Tatham \& Black, 2006).

Consistencia interna. Se evaluó la consistencia interna de las subescalas mediante dos estadísticos: el estadístico alfa de Cronbach $(\alpha)$ y el índice de fiabilidad compuesta $(f c)$. Si bien el alfa de Cronbach es uno de los indicadores más empleados en ciencias sociales (Elosua \& Zumbo, 2008), presenta algunas desventajas cuando se trabaja con variables categóricas u ordinales. Por esta razón se decidió emplear complementariamente el índice de fiabilidad compuesta, que además tiene la ventaja de contemplar las interrelaciones de los constructos extraídos cuando la escala es multifactorial. En ambos estadísticos empleados los índices superiores a .70 son considerados aceptables, mientras que aquellos que superan .80 y .90 se consideran buenos y excelentes, respectivamente. Los índices comprendidos entre .60 y .70 son considerados moderados y se sugiere no aceptar valores inferiores a estos (George \& Mallery, 2016). Los análisis estadísticos reportados fueron realizados con los programas Statistical Package for the Social Sciences (SPSS) versión 22 y Mplus 8.

\section{Resultados}

Previo a la realización del análisis factorial confirmatorio del instrumento, se llevó a cabo el análisis descriptivo de los ítems y se evaluaron los índices de asimetría y curtosis (ver Tabla 2). De la totalidad de ítems, 29 alcanzaron valores óptimos de simetría (entre .05 y .10), 12 mostraron valores aceptables (entre 1.22 y 1.96) y 9 evidenciaron valores superiores a los esperados (entre 2.12 y 3.38 ) (George \& Mallery, 2016). En lo que respecta a la curtosis, 29 ítems obtuvieron índices óptimos (entre -.91 y .95), 7 alcanzaron índices aceptables (entre 1.01 y 1.67) y los restantes mostraron índices superiores (entre 2.18 y 10.91). Lo anterior no supuso un problema, dado que el método de estimación empleado (MLR) para los análisis posteriores no requiere el cumplimiento de los supuestos de normalidad. El rango de respuesta en todos los ítems fue de 1 a 5. La mayoría de los ítems tiene una respuesta hacia la izquierda (valores cercanos a 1.5), aunque un número importante se acerca al punto medio de la distribución teórica (3). La pauta de corrección del IRISA estipula que algunos ítems deben recodificarse para calificar las subescalas, de modo que puntuaciones más elevadas en dichos ítems implican un mayor riesgo en el adolescente. 
Tabla 2.

Medias, desvíos, asimetrías y curtosis del IRISA

\begin{tabular}{|c|c|c|c|c|}
\hline Ítem & $M$ & $D E$ & Asimetría & Curtosis \\
\hline 01 & 2.36 & 1.20 & 0.66 & -0.46 \\
\hline 02 & 2.43 & 1.16 & 0.54 & -0.45 \\
\hline 03 & 1.90 & 0.78 & 0.77 & 1.01 \\
\hline 04 & 2.15 & 1.17 & 0.84 & -0.14 \\
\hline 05 & 2.04 & 1.10 & 0.99 & 0.21 \\
\hline 06 & 1.79 & 1.11 & 1.35 & 0.95 \\
\hline 07 & 2.11 & 1.01 & 0.69 & -0.20 \\
\hline 08 & 1.61 & 1.04 & 1.73 & 2.18 \\
\hline 09 & 2.22 & $1 . .00$ & 0.71 & 0.13 \\
\hline 10 & 2.39 & 1.35 & 056 & -0.91 \\
\hline 11 & 1.68 & 1.05 & 1.56 & 1.67 \\
\hline 12 & 1.62 & 1.11 & 1.79 & 2.23 \\
\hline 13 & 2.33 & 1.14 & 0.58 & -0.37 \\
\hline 14 & 2.62 & 1.13 & 0.37 & -0.50 \\
\hline 15 & 1.35 & 0.80 & 2.91 & 9.05 \\
\hline 16 & 1.62 & 1.04 & 1.81 & 2.56 \\
\hline 17 & 2.74 & 1.19 & 0.16 & -0.82 \\
\hline 18 & 2.05 & 1.02 & 0.83 & 0.19 \\
\hline 19 & 1.70 & 0.91 & 1.30 & 1.34 \\
\hline 20 & 2.49 & 1.09 & 0.42 & -0.34 \\
\hline 21 & 2.03 & 1.02 & 1.00 & 0.70 \\
\hline 22 & 2.12 & 1.20 & 0.87 & -0.19 \\
\hline 23 & 2.41 & 1.11 & 0.44 & -0.51 \\
\hline 24 & 1.95 & 1.03 & 1.22 & 1.25 \\
\hline 25 & 1.50 & 0.98 & 2.12 & 3.88 \\
\hline 26 & 1.77 & 1.31 & 1.61 & 1.18 \\
\hline 27 & 1.30 & 0.86 & 3.36 & 10.91 \\
\hline 28 & 1.55 & 1.06 & 2.15 & 3.89 \\
\hline 29 & 1.38 & 0.88 & 2.93 & 8.66 \\
\hline 30 & 1.81 & 1.07 & 1.35 & 1.21 \\
\hline 31 & 2.24 & 0.99 & 0.55 & 0.02 \\
\hline 32 & 1.53 & 1.01 & 1.95 & 2.95 \\
\hline 33 & 1.29 & 0.87 & 3.38 & 10.85 \\
\hline 34 & 1.44 & 0.93 & 2.22 & 4.22 \\
\hline 35 & 2.09 & 1.10 & 0.95 & 0.36 \\
\hline 36 & 1.89 & 0.97 & 0.79 & -0.04 \\
\hline 37 & 2.65 & 1.29 & 0.33 & -0.91 \\
\hline 38 & 2.69 & 1.22 & 0.25 & -0.85 \\
\hline 39 & 2.28 & 1.30 & 0.82 & -0.45 \\
\hline 40 & 2.19 & 1.18 & 0.72 & -0.38 \\
\hline
\end{tabular}


Continuación Tabla 2.

\begin{tabular}{ccccc}
\hline Ítem & $\boldsymbol{M}$ & $\boldsymbol{D E}$ & Asimetría & Curtosis \\
\hline 41 & 2.91 & 1.21 & 0.05 & -0.78 \\
42 & 2.44 & 1.26 & 0.45 & -0.84 \\
43 & 2.15 & 1.11 & 0.67 & -0.30 \\
44 & 1.58 & 1.04 & 1.93 & 3.02 \\
45 & 2.44 & 1.18 & 0.46 & -0.56 \\
46 & 2.22 & 1.02 & 0.75 & 0.38 \\
47 & 1.77 & 1.08 & 1.45 & 1.37 \\
48 & 2.66 & 1.28 & 0.33 & -0.90 \\
49 & 1.32 & 0.81 & 2.84 & 7.84 \\
50 & 1.29 & 0.87 & 3.30 & 10.14 \\
\hline
\end{tabular}

\section{Análisis de la estructura factorial}

La matriz de correlaciones entre los ítems indicó ausencia de colinealidad, debido a que todas las correlaciones bivariadas fueron inferiores a .85 (Cupani, 2012). Los índices de esfericidad de Bartlet $\left(\chi^{2}=10,614.231 ; p<.001\right)$ y la medida de adecuación muestral de Kaiser-Meyer-Olkin (KMO $=.95)$ indicaron apropiada relación entre variables y buena adecuación muestral. El modelo del IRISA presentó un ajuste aceptable en las cuatro dimensiones evaluadas $\left(\chi^{2} / g l=2.089, \mathrm{CFI}=.940\right.$; TLI $=$ .937; RMSEA = .050). Se inspeccionaron los pesos de regresión, resultando todos superiores a $.40, \mathrm{y}$ significativos a un nivel de $p<.001$, con excepción de los ítems 21,33, 36 y 41 . No obstante, la eliminación de estos ítems no mejoró el ajuste del modelo ni la confiabilidad de las subescalas, por lo que se decidió mantenerlos a fin de respetar la estructura original de la escala y favorecer una comparación transcultural de los datos obtenidos mediante el mismo. Respecto al ítem 41, que obtuvo la carga más baja de todo el inventario, se analizó su patrón de correlaciones con el resto de los reactivos, encontrando correlaciones significativas, aunque bajas, con 3 ítems de la subescala depresión y desesperanza, 3 ítems de la subescala ausencia de circunstancias protectoras y 11 ítems de la subescala malestar psicológico asociado al riesgo suicida, a la cual pertenece. Las correlaciones oscilaron entre $r \mathrm{~s}=-.18$ y $r \mathrm{~s}=.18$, siendo las correlaciones con los ítems del factor al que pertenece todas positivas, con excepción del ítem 18.

Las correlaciones interfactoriales fueron estadísticamente significativas y de una magnitud elevada, siendo la relación entre las subescalas de depresión y desesperanza y la de malestar psicológico asociado al riesgo suicida la que evidenció la asociación más estrecha; en la misma dirección también se identificaron correlaciones altas y moderadas entre los factores del IRISA (Figura 1). 


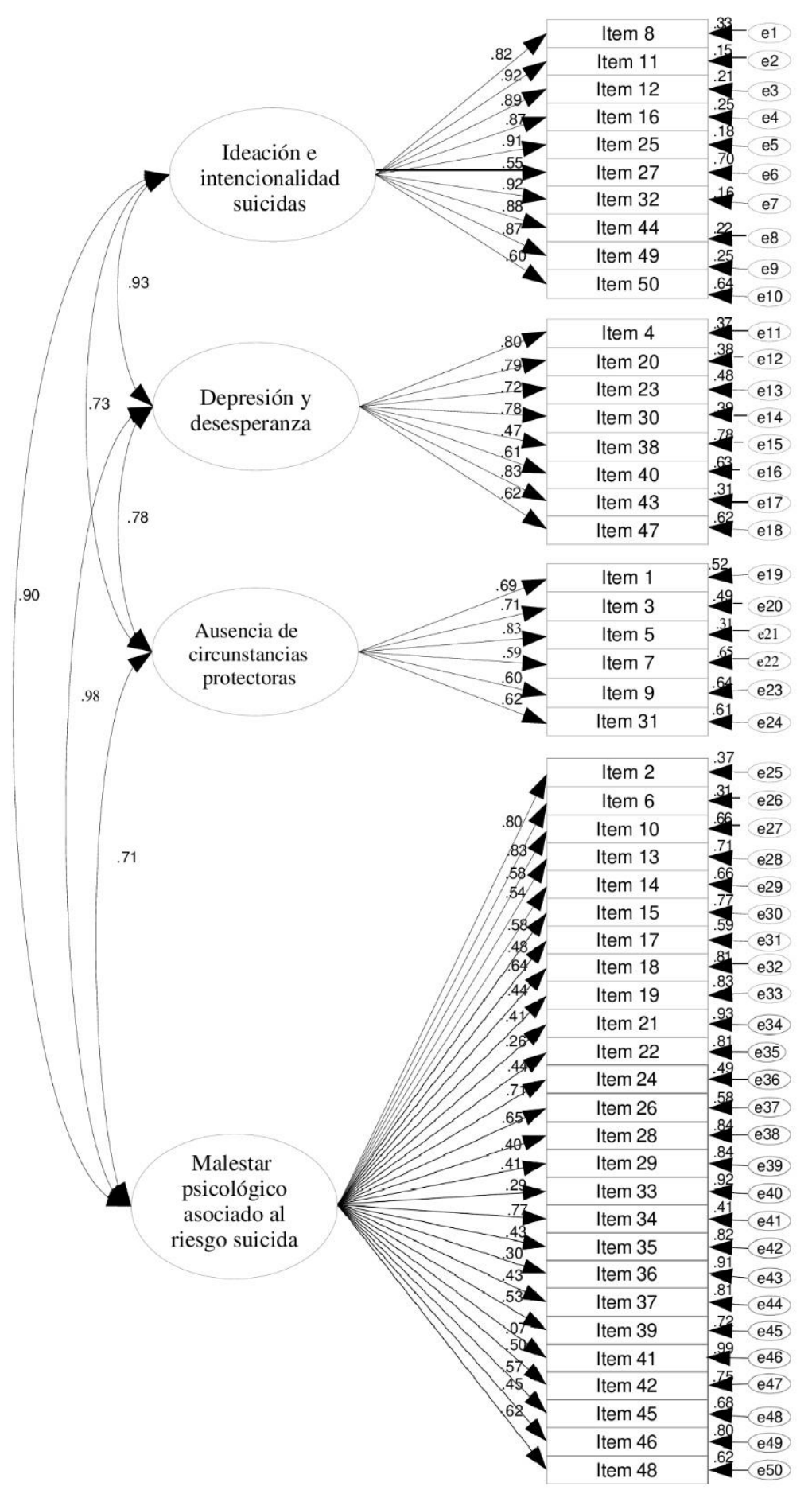

Figura 1. Modelo de cuatro factores del IRISA. 


\section{Consistencia interna}

En general, el IRISA mostró índices de fiabilidad entre buenos $(f c=.83)$ y excelentes $(f c=$ .98) para todos los factores (Tabla 3), lo que indica niveles generales adecuados de consistencia interna. Los índices de fiabilidad compuesta indicaron una mayor consistencia interna de la escala que el estadístico alfa de Cronbach $(\alpha=.80$ - .92). La $f c$ tiene como ventaja contemplar las relaciones entre los constructos extraídos cuando estos están correlacionados entre sí. Las correlaciones entre las dimensiones del instrumento indican que las subescalas ideación $e$ intencionalidad suicidas y depresión y desesperanza presentan correlaciones positivas muy altas. Ambas subescalas también mostraron correlaciones altas con la subescala malestar psicológico asociado al riesgo suicida (Figura $1)$.

Tabla 3.

Índices de consistencia interna de las subescalas del IRISA

\begin{tabular}{lcccc}
\hline Subescala & $M$ & $D E$ & $\alpha$ & $f c$ \\
\hline 1.Ideación e intencionalidad suicidas & 15.04 & 7.49 & .92 & .95 \\
2.Depresión y desesperanza & 17.70 & 6.37 & .86 & .89 \\
3.Ausencia de circunstancias protectoras & 12.88 & 4.31 & .80 & .83 \\
4.Malestar psicológico asociado al riesgo suicida & 54.50 & 14.22 & .87 & .98 \\
\hline
\end{tabular}

Nota: $\alpha=$ alfa de Cronbach. $f c=$ fiabilidad compuesta.

\section{Discusión}

La evaluación del riesgo suicida constituye un primer paso estratégico en la detección y atención primaria de jóvenes con esta problemática (OMS, 2018), y contar con herramientas validadas en muestras locales es un insumo indispensable en esta tarea. Por ello, el presente estudio se orientó a analizar algunas propiedades psicométricas, específicamente la validez basada en el contenido y la la estructura interna, y la consistencia interna del Inventario de Riesgo Suicida para Adolescentes (IRISA; Hernández-Cervantes \& Lucio, 2006) en una muestra de 447 estudiantes de institutos secundarios de Uruguay. El IRISA es uno de los pocos instrumentos específicamente diseñados para valorar el riesgo suicida en adolescentes. Ha sido empleado en muestras de México (ArenasLandgrave, Lucio-Gómez \& Forns, 2011; Córdova, Eguiluz \& Rosales, 2011; Hernández-Cervantes \& Alejandre-García, 2015; Lucio, Hernández-Cervantes \& Arenas, 2012; Lucio, Plascencia-González \& Zamarrón-Pérez, 2005), Colombia (Alarcón-Vásquez et al., 2019; Bahamón \& Alarcón-Vásquez, 2018), Paraguay (Coppari et al., 2009) y España (Ferrer \& Kirchner, 2014), aunque solamente dos de estas investigaciones se propusieron evaluar sus características psicométricas o realizar su adaptación/validación para otros contextos culturales. El presente estudio es el primero en evaluar sus propiedades psicométricas en población adolescente uruguaya.

El resultado principal de esta investigación sugiere que el IRISA (Hernández-Cervantes \& Lucio, 2006) presenta propiedades psicométricas adecuadas para evaluar el riesgo suicida en adolescentes uruguayos. Dado que el estudio original del IRISA en su segunda versión (HernándezCervantes \& Lucio, 2006) realizó un análisis factorial exploratorio y no relevó las correlaciones entre las subescalas del instrumento, a continuación, se discuten en detalle las similitudes y diferencias entre el estudio colombiano (Alarcón-Vásquez et al., 2019) y el estudio que aquí se presenta. 
Validez basada en la estructura interna: análisis factorial confirmatorio y relación entre subescalas

Respecto a la dimensionalidad del instrumento, los resultados del análisis factorial confirmatorio apoyan la constitución original de cuatro factores, obtenidos mediante un análisis factorial exploratorio con adolescentes mexicanos (Hernández-Cervantes \& Lucio, 2006). En este sentido, se apuntaría a la confirmación de la hipótesis del modelo teórico que sustenta el riesgo suicida no solamente en indicadores como la ideación, la planeación y la intencionalidad suicida, sino en potenciadores de los mismos, como la depresión y la desesperanza (Lew et al., 2019), y la ausencia de factores de protección (Valdivia, Silva, Sanhueza, Cova \& Melipillán, 2015).

Tanto en el estudio colombiano (Alarcón-Vásquez et al., 2019) como en este, los datos indican un buen nivel de ajuste al modelo propuesto por el IRISA. Si bien este estudio probó el modelo de cuatro factores, acorde al instrumento original, el estudio colombiano testeó el ajuste a tres factores, según lo propuesto para la versión abreviada. En ambos estudios se propuso la revisión de al menos un ítem con base en las bajas correlaciones ítem-subescala presentadas. A partir de los datos, se recomienda revisar cuatro reactivos de la subescala malestar psicológico asociado al riesgo suicida. Se considera que uno de ellos (21. Tengo dificultades con mis materias) podría ser altamente inespecífico del riesgo suicida, y presentar una alta comunalidad con otras dificultades prevalentes en la adolescencia, como la deserción o la discriminación escolar (Serrano-Ruiz \& Olave-Cháves, 2017). Su rol como factor de riesgo suicida podría plantearse como una interesante línea de investigación futura.

El resto de los ítems revisables (33. Si muriera me gustaría que me acompañaran [muriendo conmigo], 36. Me deshice de posesiones preciadas para mí [por ejemplo: regalando, donando, tirando, quemando] y 41. Me he reconciliado con personas con las que había tenido problemas) podrían estar evaluando componentes comunes a la planeación del intento, y específicamente en adolescentes presentar un deseo de acompañamiento/reconciliación social menor que en los adultos, considerando la búsqueda de autonomía tan relevante para esta etapa (Kawashima, Ito, Narishige, Saito \& Okubo, 2012). Si bien en este estudio dichos ítems mostraron cargas factoriales bajas, y su eliminación podría aportar a la economización del tiempo de administración, no se considera esto conveniente y se prioriza tanto el criterio teórico como la posible investigación transcultural orientada a la comparación con otros países. También se considera que cada ítem puede tener una utilidad práctica, ya que constituyen indicadores en sí mismos de aspectos emocionales, cognitivos y conductuales, que podrían integrarse en focos del plan de intervención. Esto destaca el interés clínico de los ítems como posibles ejes del trabajo psicoterapéutico con los adolescentes, aunque los mismos no estén necesariamente vinculados de manera directa al riesgo suicida, pero sí, por ejemplo, al fortalecimiento de los factores de protección detectados por el IRISA (Hernández-Cervantes \& Lucio, 2006).

En lo que respecta a la relación entre las subescalas del IRISA, y al igual que en el estudio colombiano (Alarcón-Vásquez et al., 2019), en este estudio se encontraron las correlaciones más altas entre las subescalas de ideación e intencionalidad suicidas y desesperanza y depresión, pero el segundo lugar se invierte en los estudios; mientras que para los datos del presente estudio la segunda asociación más estrecha se presentó entre depresión y desesperanza y ausencia de circunstancias protectoras, en el estudio colombiano, el segundo lugar lo ocupó la correlación entre las subescalas de ideación e intencionalidad suicidas y ausencia de circunstancias protectoras, aunque por poco margen de diferencia.

Un hallazgo llamativo común son las altas correlaciones entre las subescalas. Por un lado, esto puede derivarse del método de estimación empleado. El estimador WLSMV, aunque no asume una distribución normal de las variables manifiestas, puede producir una sobreestimación moderada de las correlaciones entre factores, cuando el tamaño de la muestra es pequeño o cuando las distribuciones 
latentes son moderadamente anormales (Li, 2016). Por otro lado, tal y como plantean AlarcónVásquez et al. (2019), la elevada correlación entre factores podría explicarse por la existencia de un constructo latente o de segundo orden más global, lo que plantea la posibilidad de que las subescalas, principalmente las de ideación e intencionalidad suicidas y depresión y desesperanza por un lado, y las de ausencia de circunstancias protectoras y malestar psicológico asociado al riesgo suicida por el otro, estén evaluando aristas muy similares del riesgo suicida. El hecho de que las subescalas de depresión y desesperanza e ideación e intencionalidad suicidas estén posiblemente solapándose en cuanto al constructo que pretenden evaluar es un fenómeno descrito en otras investigaciones sobre riesgo suicida y factores de riesgo asociados (Silva et al., 2017). Esto puede ser explicado considerando que la mayoría de las escalas de depresión incluyen ítems referentes al espectro de la conducta suicida, lo que terminaría redundando en la contribución que tienen por sí mismas la ideación/intencionalidad suicida en el continuum que culmina en el intento de suicidio propiamente dicho (Beck, Kovacs \& Weismann, 1976). Por ejemplo, en el IRISA, los reactivos: 30. Creo o siento que la gente a mi alrededor estaría mejor sin mí, 23. Me siento enojado/frustrado y 4. Creo que o siento que soy un fracaso, que pertenecen a la subescala depresión y desesperanza, apuntan a aspectos bien documentados del riesgo suicida, como la minusvalía, la sensación de no logro y la emocionalidad negativa (Federación Mundial de la Salud Mental, 2010). El contraste de un modelo de segundo orden requeriría justificación, discusiones teóricas más profundas y nuevos análisis estadísticos. Llevar esto a cabo excede los objetivos del presente trabajo. Estudios futuros podrán profundizar en esto y generar aportes en este sentido.

Cabe reflexionar, en este punto, acerca del peso semántico en la formulación de los ítems y su correlato clínico. Siguiendo el ejemplo, la palabra fracaso, no necesariamente tiene una interpretación unívoca en los jóvenes. Dichos resultados han de requerir de investigaciones más detalladas respecto a la contribución de cada uno de estos factores de riesgo suicida adolescente. Estudios futuros podrían analizar su validez convergente con instrumentos validados también en población uruguaya que, por ejemplo, evalúan específicamente ideación suicida o depresión/desesperanza, como la Escala de Desesperanza de Beck (Beck, Weissman, Lester \& Trexler, 1974) o la Escala de Ideación Suicida de Beck (Beck, Kovacs \& Weissman, 1979), considerando algunos antecedentes de su uso en este contexto y para los adolescentes uruguayos (Larrobla, Canetti, Hein, Novoa \& Durán, 2012).

\section{Consistencia interna del IRISA: fiabilidad}

Con relación a la fiabilidad, todas las subescalas del IRISA mostraron una elevada consistencia interna y homogeneidad, lo que denota una confiabilidad altamente satisfactoria. La fiabilidad de este estudio se probó evaluando tanto los índices de alfa de Cronbach como el de fiabilidad compuesta, mientras que tanto en el estudio de la primera versión del IRISA (Hernández-Cervantes \& Lucio, 2003), como en el estudio colombiano (Alarcón-Vásquez et al., 2019) analizaron exclusivamente el índice alfa de Cronbach. Tanto este estudio como los otros dos respaldan la subescala de ideación e intencionalidad suicidas como la más consistente, tomando en consideración el estadístico alfa de Cronbach. Al considerar el estadístico de fiabilidad compuesta, la subescala más consistente fue la de malestar psicológico asociado al riesgo suicida. Los altos índices de consistencia interna de esta subescala podrían deberse al elevado número de reactivos que la componen. Estudios han evidenciado que coeficientes por encima de .90 se pueden observar con regularidad en las escalas de más de 20 ítems, dado que el coeficiente se afecta en forma directa por el número de ítems que componen la escala, es decir, cuando el número de ítems es tan elevado, se sobreestima el coeficiente (CampoArias \& Oviedo, 2008). 
Una sugerencia que estudios futuros podrían considerar es la disminución de los ítems potencialmente redundantes de la subescala. A estos fines, sería de mucha utilidad un análisis con base en la Teoría de Respuesta al Ítem (TRI) para identificar aquellos indicadores con mayor sensibilidad en la valoración del riesgo suicida y evitar así la administración innecesaria de reactivos. Es importante considerar que el inventario se constituye como un instrumento de cribado, que debería apuntar a mantener, con fines prácticos, al mínimo necesario la cantidad de ítems. Si bien la eliminación de ítems limita la cantidad de información recogida, contar con una versión abreviada alternativa podría ser de utilidad para los contextos donde la economía de tiempo y recursos resulte deseable, como en atención primaria de la salud. La Organización Mundial de la Salud, junto con la Organización Panamericana de la Salud, la Junta Nacional de Drogas, el Ministerio de Salud Pública y el Ministerio de Desarrollo Social, plantean, en la II Encuesta Mundial de Salud Adolescente (2012), por ejemplo, la posibilidad de evaluar riesgo suicida en dicha población a través de pocos indicadores: uno referido a si el adolescente consideró seriamente la posibilidad de quitarse la vida, otro sobre si realizó un plan sobre cómo lo haría, y un último acerca del número de intentos de autoeliminación previos en los últimos 12 meses.

\section{Limitaciones y futuros estudios}

El estudio presenta algunas limitaciones a considerar. En primer lugar, solo se han incluido como parte de la muestra adolescentes escolarizados, lo que afectaría la representación de esta, y por lo tanto, la extrapolación de las conclusiones a otros adolescentes desvinculados del sistema educativo. Es posible que adolescentes en situaciones de alta vulnerabilidad estén sobrerepresentados entre los grupos no-escolarizados. De hecho, estudios previos cercanos geográficamente demuestran que aproximadamente la mitad de adolescentes ingresados en dispositivos de la justicia juvenil no asistía a la escuela antes del ingreso (Bobbio, 2019). Asimismo, la conducta suicida en esta población se ha mostrado significativamente superior a la de adolescentes de población general (Gallagher \& Dobrin, 2006). Por lo tanto, estudios futuros en esta población de adolescentes institucionalizados serían de sumo interés al proveer evidencia que permita mejorar la detección del riesgo suicida en esta población.

En segundo lugar, se considera recomendable analizar con detenimiento los resultados, dado que la muestra se obtuvo por conveniencia, y no resulta representativa de todos los adolescentes uruguayos. Finalmente, no se evaluó la deseabilidad social, por lo que no pueden descartarse efectos de ella en las respuestas brindadas por las personas participantes.

Futuros estudios podrían realizar un importante aporte al contrastar la validez del instrumento en otras poblaciones que pueden presentar un riesgo de conductas suicidas incrementado, como poblaciones clínicas (Barros et al., 2016) y forenses (Ford, Hartman \& Chapman, 2008). Los estudios de validez concurrente, con otras medidas empíricamente asociadas al riesgo suicida, como la depresión o la desesperanza u otras escalas breves de riesgo suicida, quedan pendientes para un futuro estudio. A su vez, estudios futuros deberían profundizar en la capacidad discriminante de cada subescala, así como revisar más detalladamente el comportamiento de los ítems que tuvieron poca contribución a sus subescalas. Además, para la realización de estudios comparativos con muestras mexicanas y colombianas, debería atenderse no solo al contraste de las puntuaciones obtenidas en el instrumento, sino a la tasa de suicidalidad prevalente en cada país. Es decir, sería esperable que, en países con mayores tasas basales de esta conducta, las puntuaciones en la población general fueran mayores. 
Finalmente, sería recomendable la inclusión de otras variables de probado interés en la conducta suicida, como la edad (adolescentes tempranos, medios y tardíos) y/o el sexo. Además, se sugiere la realización de entrevistas clínicas a parte de la muestra para identificar verdaderos positivos para el riesgo suicida, por parte de clínicos entrenados y sumar a la validez predictiva del instrumento.

\section{Implicancias de la investigación}

Pese a sus limitaciones, el estudio evidencia algunos indicios de solidez en las características psicométricas evaluadas del IRISA, que deberán seguirse estudiando y consolidando a través de futuras investigaciones. Esto permitirá la realización de estudios comparativos con otros países, por ejemplo, entre muestras mexicanas, colombianas y uruguayas, considerando que el inventario se comporta de manera similar en los tres contextos culturales. Además, este trabajo representa una de las primeras aproximaciones en el estudio sistemático del riesgo suicida en adolescentes de Uruguay, y el único que ha tenido como objetivo una primera aproximación a la adaptación de un instrumento válido y confiable para la evaluación del fenómeno.

Con relación a las implicancias teóricas, este estudio apunta a que tanto los trastornos depresivos como la desesperanza están de manera consistente y más prevalentemente asociados al riesgo suicida adolescente. La depresión está más fuertemente asociada al riesgo suicida adolescente que al adulto (Gould, Greenberg, Velting \& Shaffer, 2003), por lo que es probable que se dificulte su evaluación como una dimensión independiente del riesgo suicida en sí. Respecto a otros indicadores relevantes en el riesgo suicida adolescente, específicamente los que obtuvieron el menor peso en este estudio, podría pensarse que los mismos evalúan una dimensión del riesgo asociada al ámbito social/cultural (deseo de estar acompañados en el acto suicida, o reconciliación con personas importantes previo al suicidio); el suicidio adolescente ocurre principalmente en el contexto de búsqueda de autonomía y de conflictos sociales (familiares y de pares), por lo que podría vislumbrarse como una búsqueda de ruptura/distanciamiento vincular (Kawashima et al., 2012). Además, la planeación suicida indagada en los indicadores de menor peso en este estudio apunta a un nivel de anticipación altamente elaborado; en los adolescentes, el riesgo suicida se ha visto comúnmente vinculado a la impulsividad, en comparación con lo que ocurre en adultos (Reyna \& Farley, 2006).

En lo que refiere a las implicancias prácticas, se destaca la importancia de contar con un instrumento de valoración de riesgo suicida específico para población adolescente, tanto en los servicios de atención primaria de salud como en los contextos educativos. El IRISA se ha propuesto como un inventario de tamizaje, y como tal debería tener la ventaja de ser breve, de fácil comprensión y rápida aplicación. Los hallazgos de este estudio sugieren la posibilidad de abreviar la técnica aún más, como ya se ha realizado en otra investigación (Alarcón-Vásquez et al., 2019); es decir, se plantea la posibilidad de administrarlo como una técnica de cribado para valorar riesgo suicida adolescente en contextos con un gran flujo de casuística y que requieren evaluaciones rápidas, como los servicios de atención primaria de salud, y dejar la versión completa para su uso en contextos clínicos de atención específica de esta problemática, y a complementarse con otras medidas de variables de interés (como personalidad, autorregulación, impulsividad, conductas de riesgo, etc.). 


\section{Conclusiones}

La presente investigación permite concluir que en adolescentes uruguayos el Inventario de Riesgo Suicida presenta una buena estructura interna y una alta consistencia, y, por lo tanto, los resultados de este estudio aportan evidencia tanto sobre algunas de sus propiedades psicométricas como su adecuación en la detección de riesgo suicida en dicha población. No obstante, futuros estudios que profundicen en otras características psicométricas del instrumento, tales como su validez concurrente y predictiva, así como la posibilidad de contar con una versión más abreviada del mismo, resultarían muy beneficiosos, tanto para su aplicación en el campo de la investigación como en el contexto clínico.

\section{Financiación}

La presente investigación fue realizada gracias a la financiación de proyectos de Becas de Postgrado Nacionales, de la Agencia Nacional de Investigación e Innovación (ANII).

\section{Referencias}

Alarcón-Vásquez, Y., Bahamón, M., Trejos-Herrera, A. M., González-Gutiérrez, O., Rubio-Castro, R., Hernández-Cervantes, Q. et al. (2019). Análisis de las Propiedades Psicométricas del Inventario de Riesgo Suicida (IRISA) para Adolescentes Colombianos. Revista Iberoamericana de Diagnóstico y Evaluación - e Avaliação Psicológica, 51(2), 87-98.

Ardiles-Irarrázabal, R. A., Alfaro-Robles, P. A., Díaz-Mancilla, I. E. \& Martínez-Guzmán, V. V. (2018). Riesgo de suicidio adolescente en localidades urbanas y rurales por género, región de Coquimbo, Chile. Aquichan, 18(2), 160-170.

Arenas-Landgrave, P., Lucio-Gómez, E. \& Forns, M. (2011). Indicadores diferenciales de personalidad frente al riesgo de suicidio en adolescentes. RIDEP, 2(32), 79-104.

Bahamón, M. J. \& Alarcón-Vásquez, Y. (2018). Diseño y validación de una escala para evaluar el Riesgo Suicida (ERS) en adolescentes colombianos. Universitas Psychologica, 17(4), 1-15.

Barros, J., Morales, S., Echávarri, O., García, A., Ortega, J., Asahi, T., et al. (2016). Suicide detection in Chile: proposing a predictive model for suicide risk in a clinical sample of patients with mood disorders. Brazilian Journal of Psychiatry, 39(1), 1-11.

Beck, A. T., Kovacs, M. \& Weissman, A. (1976). Hopelessness and suicidal behavior, JAMA, 234, 1146-1149.

Beck, A. T., Kovacs, M. \& Weissman, A. (1979). Assessment of suicidal ideation. The scale for Suicide Ideation. Journal of Consulting Clinical Psychology, 47(2), 343-352.

Beck, A. T., Weissman, A., Lester, D. \& Trexler, L. (1974). The measurement of pessimism: The Hopelessness Scale. Journal of Consulting and Clinical Psychology, 42(6), 861-865.

Bobbio, A. (2019). Conducta antisocial adolescente: Un contraste empírico de teorías criminológicas. (Disertación doctoral). Universidad Nacional de Córdoba, Argentina.

Brás, M., Carmo, C. \& Neves de Jesus, S. (2017). Estudo das propriedades psicométricas do Inventário de Reconhecimento de Sinais de Alerta para Atos Suicidas. Revista Iberoamericana de Diagnóstico y Evaluación - e Avaliação Psicológica, 1(43), 89-105.

Campo-Arias, A. \& Oviedo, H. C. (2008). Propiedades psicométricas de una escala: la consistencia interna. Revista de salud pública, 10, 831-839. 
Coppari, N., Alonso R., Fernández, E., Iturry, V., Kriskovich, R. \& Mernes, R. (2009). Adolescencia prevenida: diferencias en indicadores de riesgo en adolescentes chaqueños medidas a través del IRIS. Eureka, 6(1), 58-75.

Córdova, M., Eguiluz, L. \& Rosales, J. (2011). Pensamientos suicidas en estudiantes universitarios del estado de Tlaxcala (México). Enseñanza e investigación en psicología, 16(1), 155-164.

Cupani, M. (2012). Análisis de ecuaciones estructurales: Conceptos, etapas de desarrollo y un ejemplo de aplicación. Revista Tesis, 1, 186-199.

Echávarri, O., Morales, S., Barros, J., Armijo, I., Larraza, D., Longo, A., et al. (2018). Validación de la Escala Razones Para Vivir en Consultantes a Salud Mental de la Región Metropolitana de Chile. Psykhe, 27(2), 1-17.

Elosua, P. \& Zumbo, B. D. (2008). Coeficientes de fiabilidad para escalas de respuesta categórica ordenada. Psichothema, 20(4), 896-901.

Federación Mundial de la Salud Mental. (2010). Enfermedad mental y suicidio. Guía para la familia para encarar y reducir riesgos. Woodbridge, VA: World Federation for Mental Health.

Fernández-Liporace, M. \& Casullo, M. M. (2006). Validación factorial de una escala para evaluar riesgo suicida. Revista Iberoamericana de Diagnóstico y Evaluación Psicológica, 21(1), 922.

Ferrer, L. \& Kirchner, T. (2014). Suicidal tendency in a sample of adolescent outpatients with Adjustment Disorder: Gender differences. Comprehensive Psychiatry, 55, 1342-1349.

Ford, J. D., Hartman, J. K., Hawke, J. \& Chapman, J. F. (2008). Traumatic victimization, posttraumatic stress disorder, suicidal ideation, and substance abuse risk among juvenile justice-involved youth. Journal of Child \& Adolescent Trauma, 1(1), 75-92.

Freiberg-Hoffmann, A., Stover, J. B., de la Iglesia, G. \& Fernández-Liporace, M. (2013). Correlaciones policóricas y tetracóricas en estudios factoriales exploratorios y confirmatorios. Ciencias Psicológicas, 7(2), 151-164.

Galarza, A., Castañeiras, C. \& Fernández, M. (2014). Construcción y estudio de calidad psicométrica de la Escala Argentina de Suicidabilidad para Adolescentes (EASA). Anuario de Proyectos e Informes de Becarios de Investigación, 11, 1009-1016.

Gallagher, C. A. \& Dobrin, A. (2006). Deaths in juvenile justice residential facilities. Journal of Adolescent Health, 38(6), 662-668.

George, D. \& Mallery, P. (2016). IBM SPSS statistics 23 step by step: A simple guide and reference. Recuperado de https://books.google.co.kr/books?hl=en\&lr= \&id=vKLOCwAAQBAJ\&oi=fnd\&pg=PP1\&dq=D.+George,+P.+Mallery,+IBM+SPSS+ Statistics+23+Step+by+Step:+A+Simple+Guide+and+Reference,+Routledge,+

González-Macip, S., Díaz, A., Ortiz, S., González, C. \& González, J. J. (2000). Características psicométricas de la Escala de Ideación Suicida de Beck (ISB) en estudiantes universitarios de la ciudad de México, Salud Mental, 23(2), 21-30.

Gould, M. S., Greenberg, T., Velting, D. M. \& Shaffer, D. (2003). Youth suicide risk and preventive interventions: a review of the past 10 years. Journal of the American Academy of Child \& Adolescent Psychiatry, 42(4), 386-405.

Hair, J. F., Anderson, R. E., Tatham, R. L. \& Black, W. (2006). Análisis multivariante (6a. ed.). Madrid, España: Pearson Education.

Hernández-Cervantes, Q. \& Alejandre-García, J. (2015). Relación entre riesgo suicida y uso de tabaco o alcohol en una muestra de universitarios. Acta universitaria, 25(2), 47-51.

Hernández-Cervantes, Q. \& Lucio, E. (2003). Inventario de riesgo suicida para adolescentes IRIS, primera versión. Reporte del examen de postulación al doctorado, Facultad de Psicología, Universidad Nacional Autónoma de México, México. 
Hernández-Cervantes, Q. \& Lucio, E. (2006). Evaluación del riesgo suicida y estrés asociado en adolescentes estudiantes mexicanos. Revista Mexicana de Psicología, 23(1), 45-52.

Hernández-Cervantes, Q. \& Lucio, E. (2010). Modelo de evaluación del riesgo suicida adolescente basado en la exposición a eventos estresantes y configuraciones de personalidad. Recuperado de https://www.researchgate.net/publication/291523665

Kawashima, Y., Ito, T., Narishige, R., Saito, T. \& Okubo, Y. (2012). The characteristics of serious suicide attempters in Japanese adolescents - comparison study between adolescents and adults. BMC Psychiatry, 12, 191-198.

King, J. D. \& Kowalchuk, B. (1994). ISO-30. Adolescent Inventory of Suicide Orientation-30. Minneapolis: National Computer Systems.

Larrobla, C., Canetti, A., Hein, P., Novoa, G. \& Durán, M. (2012). Prevención de la conducta suicida en adolescentes: Guía para los sectores Educación y Salud. Montevideo: Udelar-CSIC.

Lew, B., Huen, J., Yu, P., Yuan, L., Wang, D.F., Ping, F., et al. (2019). Associations between depression, anxiety, stress, hopelessness, subjective well-being, coping styles and suicide in Chinese university students. PLoS ONE, 14(7), 1-10.

Li, CH. (2016). Confirmatory factor analysis with ordinal data: Comparing robust maximum likelihood and diagonally weighted least squares. Behav Res Methods, 48(3), 936-49. doi: https://doi.org/10.3758/s13428-015-0619-7.

Lloret-Segura, S., Ferreres-Traver, A., Hernández-Baeza, A. \& Tomás-Marco, I. (2014). El análisis factorial exploratorio de los ítems: Una guía práctica, revisada y actualizada. Anales de Psicología, 30(3), 1151-1169. doi: https://doi.org/10.6018/analesps.30.3.199361

Lucio, E., Hernández-Cervantes, Q. \& Arenas, P. (2012). Modelo de tamizaje para uso de sustancias y riesgo suicida en estudiantes de bachillerato: resultados preliminares. En L. M. Reidl, Desarrollo de nuevos modelos para la prevención y el tratamiento de conductas adictivas. Seminarios académicos (pp.133-146). México DF: Porrúa / UNAM.

Lucio, E., Loza, C. G. \& Durán P. C. (2000). Los sucesos de vida estresantes y la personalidad de los adolescentes con intento suicida. Revista de Psicología Contemporánea, 7(2), 58-65.

Lucio, E., Plascencia-González, M. \& Zamarrón-Pérez, G. (2005). Detección del riesgo suicida y otros problemas emocionales en una muestra de adolescentes de Aguascalientes. Investigación y Ciencia, 13(32), 29-36.

Machado, A. I. (2006). Detección de riesgo suicida en una secundaria pública del Distrito Federal. (Tesis de licenciatura inédita). Facultad de Psicología, Universidad Nacional Autónoma de México, México.

Ministerio de Salud Pública, Fondo de Población de Naciones Unidas. (2015). Aportes para el abordaje de la salud de adolescentes en el Primer Nivel. Recuperado de http://www.msp.gub.uy/sites/default/files/archivos_adjuntos/Publicacion\%20Adolescentes.p df

Ministerio de Salud Pública. (2013, julio). Suicidio en adolescentes y jóvenes de 10 a 24 años en Uruguay, 2009. Programa Nacional de Salud Mental, Programa Nacional de Salud de Adolescencia y Juventud. Documento presentado en el Día Nacional de Prevención del Suicidio, Montevideo, Uruguay.

Ministerio de Salud Pública. (2018). Índice de suicidios se mantuvo estable en 2018, 20,25 por cada 100.000 habitantes. Recuperado de https://www.gub.uy/ministerio-saludpublica/comunicacion/noticias/indice-suicidios-se-mantuvo-estable-2018-2025-cada100000-habitantes

Miron, O., Yu, K. H., Wilf-Miron, R. \& Kohane, I. S. (2019). Suicide rates among adolescents and young adults in the United States, 2000-1017. JAMA, 321(23), 2362-2364. 
Organización Mundial de la Salud. (2012). Prevención de suicidio. Recuperado de http://www.who.int/ mediacentre/news/releases.

Organización Mundial de la Salud. (2014). Primer informe de la OMS sobre prevención del Suicidio. Recuperado de https://www.who.int/mediacentre/news/releases/2014/suicide-preventionreport/es/

Organización Mundial de la Salud. (2018). Suicide rate estimates, age-standardized: Estimates by country. $\quad$ Recuperado

https://apps.who.int/gho/data/node.main.MHSUICIDEASDR?lang=en

Organización Mundial de la Salud. (2018). Suicidio. Recuperado de https://www.who.int/es/newsroom/fact-sheets/detail/suicide

Organización Mundial de la Salud. (2019). Suicidio: datos y cifras. Recuperado de https://www.who.int/es/news-room/fact-sheets/detail/suicide

Organización Panamericana de la Salud, Organización Mundial de la Salud, Junta Nacional de Drogas, Ministerio de Salud Pública \& Ministerio de Desarrollo Social. (2012). Adolescencias, un mundo de preguntas - II Encuesta Mundial de Salud Adolescente GSHS 2012. Montevideo: OPS, JND, MSP y Mides.

Organización Panamericana de la Salud. (2014). Mortalidad por suicidio en las Américas: Informe regional. Washington DC: OPS.

Quinlan-Davidson, M., Sanhueza, A., Espinosa, I., Escamilla-Cejudo, J.A. \& Maddaleno, M. (2014). Suicide among young people in the Americas. Journal of Adolescent Health, 54(3), 262-268.

Reyna, V. \& Farley, F. (2006). Risk and rationality in adolescent decision-making: implications for theory, practice, and public policy. Psychological Science in the Public Interest, 7(1), 1-44.

Sarchiapone, M., D’Aulerio, M. \& Iosue, M. (2015). Suicidal ideation, suicide attempts and completed suicide in adolescents: Neuro-biological aspects. Biological Aspects of Suicidal Behavior, 30, 11-20.

Serrani-Azcurri, D. (2017). Psychometric validation of the Columbia-Suicide Severity rating scale in Spanish-speaking adolescents. Colombia Médica, 48(4), 174-182.

Serrano-Ruiz, C. \& Olave-Cháves, J. (2017). Factores de riesgo asociados con la aparición de conductas suicidas en adolescentes. Med UNAB, 20(2), 139-147.

Shaffer, D. \& Pfeffer, C. R. (2001). Practice parameter for the assessment and treatment of children and adolescents with suicidal behavior. Journal of the American Academy of Child \& Adolescent Psychiatry, 40(Supl. 7), 24S-51S.

Silva, D., Valdivia, M., Vicente, B., Arévalo, E., Dapelo, R. \& Soto, C. (2017). Intento de suicidio y factores de riesgo en una muestra de adolescentes escolarizados en Chile. Revista de Psicopatología y Psicología Clínica, 22, 33-42.

Valdéz, L. (2010). Prestaciones Psicoterapéuticas, Adultos y niños, adolescentes y jóvenes. Uruguay: MSP, PNSM.

Valdivia, M., Silva, D., Sanhueza, F., Cova, F. \& Melipillán, F. (2015). Prevalencia de intento de suicidio adolescente y factores de riesgo asociados en una comuna rural de la provincia de Concepción. Revista Médica de Chile, 143(3), 320-328.

Villacieros, M., Bermejo, J., Magaña, M. \& Fernández-Quijano, I. (2016). Psychometric properties of the Attitudinal Beliefs Questionnaire about suicidal behavior (CCCS-18). The Spanish Journal of Psychology, 19, E68. doi: doi.org/10.10 17/sjp.2016.68

Villalobos-Galvis, F. H. (2010). Validez y fiabilidad del Inventario de Ideación Suicida Positiva y Negativa-PANSI, en estudiantes colombianos. Universitas Psychologica, 9(2), 509-520. 
Villalobos-Galvis, F. H., Arévalo Ojeda, C. \& Rojas Rivera, F. D. (2012). Adaptación del Inventario de Resiliencia ante el Suicidio (SRI-25) en adolescentes y jóvenes de Colombia. Revista Panamericana de Salud Pública, 31(3), 233-239.

Contribución de los autores: a) Concepción y diseño del trabajo; b) Adquisición de datos; c) Análisis e interpretación de datos; d) Redacción del manuscrito; e) revisión crítica del manuscrito.

A. I. M. ha contribuido en a, b, c, d, e; A. B. ha contribuido en c, d, e; K. A. ha contribuido en c, d, e; A. P. ha contribuido en b, c, d, e; C. R. ha contribuido en d, e; Q. H. C. ha contribuido en d, e.

Editora científica responsable: Dra. Cecilia Cracco. 